\title{
Mitochondrial Transmembrane Potential and Reactive Oxygen Species Generation Regulate the Enhanced Effect of CCCP on TRAIL-Induced SNU-638 Cell Apoptosis
}

\author{
Atul A. CHAUDHARI ${ }^{1)}$, Jae-Won SEOL ${ }^{1)}$, Seog-Jin $\mathrm{KANG}^{2)}$ and Sang-Youel PARK ${ }^{1) *}$ \\ ${ }^{1)}$ Center for Healthcare Technology Development, College of Veterinary Medicine, Bio-Safety Research Institute, Chonbuk National \\ University, Jeonju, Jeonbuk 561-756 and ${ }^{2}$ National Institute of Animal Science, Cheonan 330-801, South Korea
}

(Received 27 November 2006/Accepted 21 January 2008)

\begin{abstract}
TRAIL is a member of the tumor necrosis factor family and engages apoptosis via recruitment and rapid activation of caspase8. This study investigated the effect of carbonyl cyanide $m$-chlorophenylhydrazone (CCCP), a classic uncoupler of oxidative phosphorylation, on TRAIL-induced apoptosis in SNU-638 cells derived from human gastric cancer cells. It was found that treatment with CCCP followed by incubation with TRAIL markedly enhanced apoptosis by 2 fold compared with treatment with TRAIL alone. This effect was accompanied by reduction in mitochondrial transmembrane potential and generation of reactive oxygen species. This sensitization was inhibited by $N$-acetyl-l-cysteine, which restored the mitochondrial transmembrane potential and reduced reactive oxygen species generation. Treatment with $N$-acetyl-L-cysteine also inhibited expression of apoptotic proteins such as Bax and Smac and abrogated caspase8 activation. Moreover, treatment with $\mathrm{N}$-acetyl-L-cysteine prior to induction with TRAIL increased expression of the anti-apoptotic Bcl2 protein. These data indicate that CCCP enhanced TRAIL-induced apoptosis by dissipation of mitochondrial transmembrane potential and reactive oxygen species, suggesting that treatment with CCCP combined with that with TRAIL can be an efficient method to induce death of tumor cells, particularly cells that are resistant to TRAIL-induced apoptosis.
\end{abstract}

KEY WORDS: caspase, CCCP, reactive oxygen species, TRAIL.

Apoptosis is a tightly regulated cell death program that plays a key role in a variety of biological processes. Unlike necrosis, apoptosis is a fundamental biochemical process that involves selective and controlled elimination of cells in multicellular organisms during normal cellular differentiation and development $[5,20,24]$. Apoptosis is characterized by membrane blebbing, nuclear fragmentation and formation of apoptosomes and is involved in tissue homeostasis, aging, various pathological processes and irreversible cell injury [9, 20]. Regulation of apoptosis occurs through extrinsic and intrinsic pathways. The extrinsic pathway is engaged through the so-called death receptors on the cell surface $[2,3,15]$. Mitochondria play an important role in the intrinsic pathway. Misregulation or failure of apoptosis can result in development of cancer, and resistance to antitumor treatment might be due to the insensitivity of cancer cells to induction of apoptosis. Therefore, apoptosis is a mechanism that needs to be exploited when developing new chemotherapies for cancer.

Carbonyl cyanide $m$-chlorophenylhydrazone (CCCP) is a protonophore that renders the mitochondrial inner membrane permeable to protons and causes dissipation of the proton gradient across the inner mitochondrial membrane. CCCP also causes uncoupling of mitochondria, which means that transfer of electrons through the electron transfer chain is no longer linked to production of adenosine triphosphate due to loss of the electrochemical gradient. CCCPinduced apoptosis has been reported in many cell lines,

\footnotetext{
* Correspondence to: Park, S.-Y., College of Veterinary Medicine, Chonbuk National University, Jeonju, Jeonbuk 561-756, South Korea. e-mail: sypark@chonbuk.ac.kr.
}

including Jurkat-neo, the FL5.12 cell line, HL-60 and ST486 [4, 13, 14, 17]. However, some studies have reported that CCCP alone does not induce apoptosis [7, 8, 12].

TRAIL (tumor necrosis factor-related apoptosis-inducing ligand) is a recently identified member of the TNF family that also activates the cognate receptor molecules through a trimerization process that is similar to other members of the TNF family [19, 23]. TRAIL has unique selectivity for triggering apoptosis in tumor cells by engaging apoptosis via recruitment and rapid activation of caspase- 8 and might be less active against normal cells. However, the role of reactive oxygen species (ROS) in TRAIL-induced apoptosis is unclear. Recent preclinical studies have demonstrated that repeated systemic administration of the recombinant TRAIL protein effectively limits tumor growth without serious side effects $[6,22]$. Therefore, TRAIL has attracted considerable attention as a promising cancer treatment.

Mitochondria play an important role in the intrinsic pathway of apoptosis by transmitting the cell death signals to the cytosol, which leads to activation of caspases. A number of changes in mitochondria occur after activation of the apoptotic signals, such as a loss of mitochondrial membrane potential $(\Delta \Psi \mathrm{m})$, generation of ROS, opening of the permeability transition pore and release of apoptotic factors into the cytosol $[10,16]$. In this study, we examined the effect of mitochondrial uncoupling on TRAIL-induced apoptosis in SNU-638 cells. The results showed that this uncoupling effect enhanced TRAIL-induced apoptosis in SNU-638 cells by loss $\Delta \Psi \mathrm{m}$ and ROS generation and subsequently release apoptotic factors from the mitochondria. Therefore, we report that loss of $\Delta \Psi \mathrm{m}$ and ROS generation regulates enhancement of TRAIL-induced apoptosis by CCCP. 


\section{MATERIALS AND METHODS}

Cell culture and reagents: SNU-638 (human gastric cancer) cells were obtained from the Korea Cell Line Bank (Seoul, Korea) and were maintained in RPMI 1640 culture medium supplemented with $10 \%(\mathrm{v} / \mathrm{v})$ fetal bovine serum and antibiotics $(100 \mathrm{U} / \mathrm{m} l$ penicillin and $100 \mu \mathrm{g} / \mathrm{m} l$ streptomycin). The cell cultures were incubated in an atmosphere containing $5 \% \mathrm{CO}_{2}$ at $37^{\circ} \mathrm{C}$. The $\mathrm{CCCP}$ was dissolved in dimethylsulfoxide at a concentration of $20 \mathrm{mM}$ and was used at a final concentration of 5-80 $\mu \mathrm{M}$. CCCP and $N$ acetyl-L-cysteine (NAC) were purchased from the Sigma Chemical Co. (St. Louis, MO, U.S.A.), and 2', 7'-Dichlorofluorescin diacetate and 5,5',6,6'-tetrachloro-1,1',3,3'tetraethylbenzimidazol-carbocyanine iodide were purchased from Molecular Probes (Eugene, OR, U.S.A.).

Cell viability test: The SNU-638 cells were plated at $1.0 \times$ $10^{4}$ cells in a 12 -well plate and incubated at $37^{\circ} \mathrm{C}$ for $24 \mathrm{hr}$. The cells were pretreated with different doses $(0,5,10,20$, 40 , and $80 \mu \mathrm{M})$ of CCCP for 6 hrs and subsequently treated with recombinant TRAIL protein [21] for $3 \mathrm{hr}$. After treatment with CCCP $(20 \mu \mathrm{M})$ for different periods of time $(0,1$, $3,6$ and $12 \mathrm{hr})$, the recombinant TRAIL protein $(100 \mathrm{ng} / \mathrm{ml})$ was added to the culture media and incubated for another 3 $\mathrm{hr}$. The cells were also treated with NAC (1 mM) for $1 \mathrm{hr}$, and this was followed by CCCP $(20 \mu \mathrm{M})$ for $6 \mathrm{hr}$ and TRAIL $(100 \mathrm{ng} / \mathrm{ml})$ for an additional $3 \mathrm{hr}$. The cell morphology was photographed using a camera attached to an optical microscope, and the cell viability was determined using the crystal violet staining method, as described elsewhere [18].

Evaluation of mitochondrial transmembrane potential $(\Delta \Psi m)$ : The level of mitochondrial energization was determined using a lipophilic cation, 5,5',6,6'-Tetrachloro1,1',3,3'-tetraethylbenzimidazol-carbocyanine iodide. This method is based on the ability of a fluorescent probe to enter mitochondria selectively and reversibly change its color from green to orange with increasing mitochondrial potential. Briefly, the cells were treated with NAC (1 mM), CCCP $(20 \mu \mathrm{M})$ and TRAIL $(100 \mathrm{ng} / \mathrm{m} l)$ for the indicated time and manner. The cells were then collected by centrifugation, washed twice with phosphate-buffered saline (PBS) and resuspended in $500 \mathrm{~m} l$ PBS containing 5,5',6,6'-Tetrachloro-1,1',3,3'-tetraethylbenzimidazol-carbocyanine iodide at a concentration of $10 \mathrm{mM}$. After incubation for 30 min at $37^{\circ} \mathrm{C}$, the cells were photographed using a fluoroscope.

Determination of ROS: The non-fluorescent probe 2',7'Dichlorofluorescein diacetate, which converts to highly fluorescent 2',7'-dichlorofluorescein after a reaction with ROS, was used to monitor the production of ROS. Briefly, after treatment, the media was collected in a 96-well microplate, and diluted 2',7'-Dichlorofluorescein diacetate at a concentration of $10 \mu \mathrm{M}$ was added to each well. After incubation for $30 \mathrm{~min}$ at $37^{\circ} \mathrm{C}$, the fluorescence was determined using a fluorescence plate reader (SpectraMax M2, Molecular Devices, Sunnyvale, CA, U.S.A.) at an excitation and emission wavelength of $490 \mathrm{~nm}$ and $525 \mathrm{~nm}$, respectively.
Western blot assay: Whole cell lysates were prepared by harvesting the cells, washing them in cold PBS, resuspending them in a lysis buffer [25 mM Hepes ( $\mathrm{pH} 7.4), 100 \mathrm{mM}$ EDTA, $5 \mathrm{mM} \mathrm{MgCl}_{2}, 0.1 \mathrm{mM}$ Dithiothreitol, and protease inhibitor cocktails (Roche Diagnostics, Mannheim, Germany) and subjecting then to sonication. The proteins (30 $\mu \mathrm{g} / \mathrm{m} l)$ were separated on a 12 to $15 \%$ SDS gel and transferred to a nitrocellulose membrane. After incubation with primary and secondary antibodies, the membranes were developed by enhanced chemiluminescence. Protein expression was tested using antibodies against human caspase- 8 and Bax (both from BD Pharmingen, San Diego, CA, U.S.A.) and Bcl-2 and Smac (both from Santa Cruz Biotech, Santa Cruz, CA, U.S.A.).

\section{RESULTS}

Enhancement of TRAIL-induced apoptosis by CCCP treatment: To examine the effect of CCCP on TRAILinduced cell death in SNU-638 cell, human gastric cancer cells were treated with the indicated doses and times and then with the TRAIL protein for an additional $3 \mathrm{hr}$. As shown in Fig. 1, CCCP enhanced TRAIL-induced apoptosis in a dose- and time-dependent manner. TRAIL alone induced only $20 \%$ cell death, while CCCP markedly enhanced the TRAIL effect for different concentrations and different time periods (Figs. 1A and 1B). Although the enhanced effect of CCCP was less at low concentrations (5 and $10 \mu \mathrm{M}), \mathrm{CCCP}$ at $20 \mu \mathrm{M}$ enhanced TRAIL-induced apoptosis by $60 \%$ compared with treatment with TRAIL alone. The level of cell death was increased by almost threeto four-fold with higher doses (Fig. 1A). The cell morphology also supported the enhanced effect of CCCP and showed that CCCP treatment at all doses increased the number of apoptotic cells compared with that of CCCP and TRAIL alone (Fig. 1C). In regard to the different time periods, TRAIL-induced apoptosis was enhanced 2- to 3-fold after treatment with CCCP for 6 and $12 \mathrm{hr}$, respectively (Fig. 1B). These results showed that treatment with CCCP sensitized the SNU-638 cells to TRAIL-induced apoptosis and suggest that treatment with CCCP enhances the effect of TRAIL in a time- and dose-dependent manner in SNU-638 cells.

CCCP-induced loss of $\triangle \Psi m$ and ROS production enhanced TRAIL-induced apoptosis in the SNU-638 cell line: To determine if dissipation of $\Delta \Psi \mathrm{m}$ and generation of ROS are responsible for the enhanced effect of CCCP on TRAIL-induced apoptosis in SNU-638 cells, we used NAC as an ROS scavenger. The cells were treated with NAC for $1 \mathrm{hr}$ before a 6-hour CCCP treatment and were subsequently treated with TRAIL for an additional $3 \mathrm{hr}$. Figure 2A clearly shows that the NAC treatment inhibited the enhanced effect of CCCP on TRAIL induced apoptosis. CCCP enhanced the TRAIL effect and reduced the cell viability by $70 \%$ compared with that of the negative control, while NAC treatment restored the cell viability to $70 \%$ (Fig. $2 \mathrm{~A}$ ). In order to elucidate the mechanism involved in the enhanced apoptotic 
A

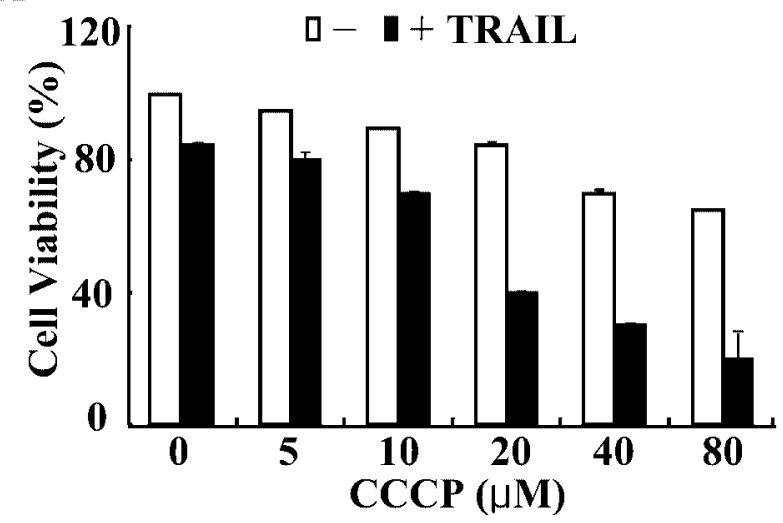

B

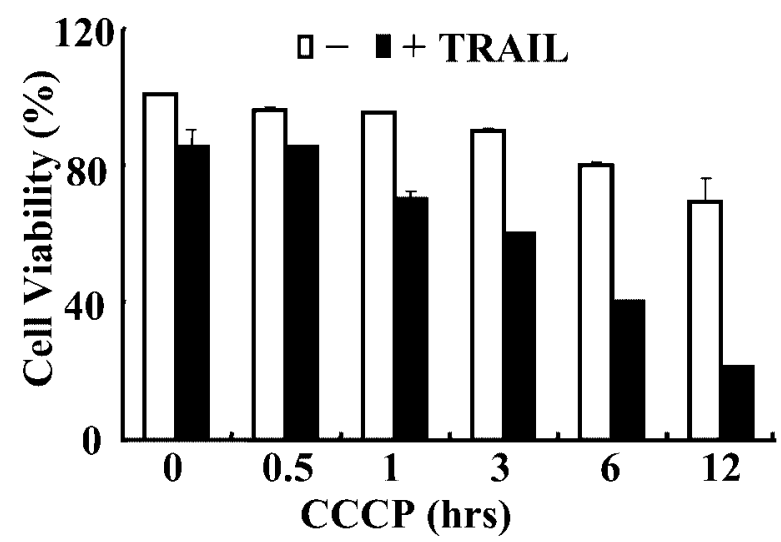

C

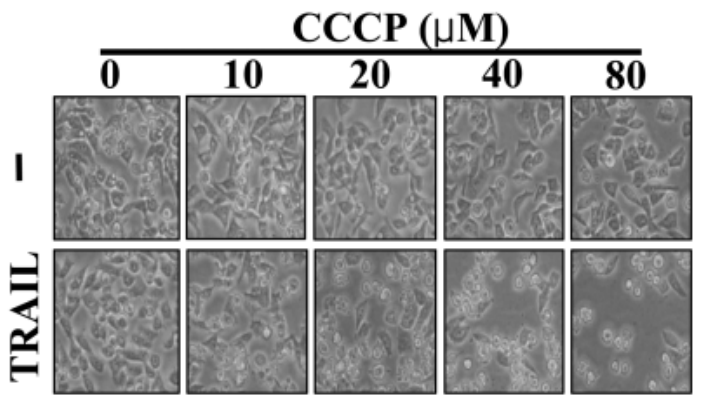

Fig. 1. CCCP enhances TRAIL-induced apoptosis in a doseand time-dependent manner.(A) SNU-638 cells plated on a 12-well plate were treated with CCCP $(6 \mathrm{hr})$ at the indicated concentrations followed by TRAIL $(100 n \mathrm{~g} / \mathrm{m} l)$ treatment for an additional $3 \mathrm{hr}$. Cell viability was determined by crystal violet staining method. Viability of control cells was set at $100 \%$, and viability relative to the control was presented. The experiments were performed in triplicate, at least twice. The bar indicates the standard error. (B) SNU-638 cells were treated with CCCP $(20 \mu \mathrm{M})$ for the indicated time periods and then with or without TRAIL $(100 n \mathrm{~g} / \mathrm{m} l)$ protein for $3 \mathrm{hr}$. Cell viability was determined as described above. (C) Cell morphology under the conditions described in A was photographed $(\times 200)$.

effect of the CCCP treatment, $\triangle \Psi \mathrm{m}$ was measured using the 5,5',6,6'f-Tetrachloro-1,1',3,3'-tetraethylbenzimidazol-carbocyanine iodide probe, as described in materials and methods. The photomicrographs indicate that the CCCP treatment and combined treatment with CCCP and TRAIL caused significant dissipation of $\Delta \Psi \mathrm{m}$ compared with that of the negative control (Fig. 2B). When the mitochondrial transmembrane potential is high, 5,5',6,6'-Tetrachloro1,1',3,3'-tetraethylbenzimidazol-carbocyanine iodide dye produced an aggregate and red fluorescence; green fluorescence is produced when $\Delta \Psi \mathrm{m}$ is low. The fluoroscopic results presented in Fig. 2B show green fluorescence in cells treated with $\mathrm{CCCP}$ and the combined treatment with $\mathrm{CCCP}$ and TRAIL protein, indicating lower $\Delta \Psi \mathrm{m}$ values. In contrast, the negative control cells and cells treated with TRAIL alone showed red fluorescence, indicating high $\Delta \Psi \mathrm{m}$ values. Treatment with NAC restored $\Delta \Psi \mathrm{m}$ values lowered by treatment with CCCP (Fig. 2B), indicating that NAC might be involved in inhibiting the dissipation of $\triangle \Psi \mathrm{m}$ by treatment with CCCP. In order to test the role of ROS in TRAILinduced apoptosis, ROS generation in the presence of CCCP was examined using 2',7'-Dichlorofluorescein fluorescence. CCCP alone induced ROS generation compared with the control and TRAIL alone. On the other hand, the cells treated with CCCP and then TRAIL showed an increase in
ROS generation (Fig. 2C). Conversely, the NAC treatment blocked generation of ROS in the cells treated with CCCP alone and CCCP with TRAIL and prevented the enhancing effect of CCCP on TRAIL-induced apoptosis by restoring cell viability (Figs. $2 \mathrm{~A}$ and $2 \mathrm{C}$ ). Thus, all together, these data suggest that CCCP enhanced TRAIL-induced apoptosis by reducing $\triangle \Psi \mathrm{m}$ and ROS generation.

Antioxidant NAC regulates the expression levels of Bax, Smac, Bcl-2 and active caspase- 8 proteins: The expression levels of Bax, Smac, caspase- 8 and anti-apoptotic protein Bcl-2 were examined in order to gain some insight into the apoptotic proteins involved. As shown in Fig. 3, the CCCP treatment with TRAIL increased activation of the Bax and Smac proteins. CCCP alone also induced activation of these proteins, but to a lesser extent than the combined CCCP and TRAIL treatment. The NAC treatment abrogated the activation of these mitochondrial related proteins, which corresponds to the results concerning $\Delta \Psi \mathrm{m}$ and ROS generation (Figs. 2B and 2C). This suggests that NAC inhibits dissipation of $\triangle \Psi \mathrm{m}$ and ROS generation and thereby prevents activation of these apoptotic proteins.

The CCCP treatment also enhanced the TRAIL-induced activation of caspase- 8 , which is dependent on ROS generation. The cells treated with CCCP alone expressed Bcl-2, and NAC treatment reexpressed Bcl-2 protein in $\mathrm{CCCP}$ and 
A

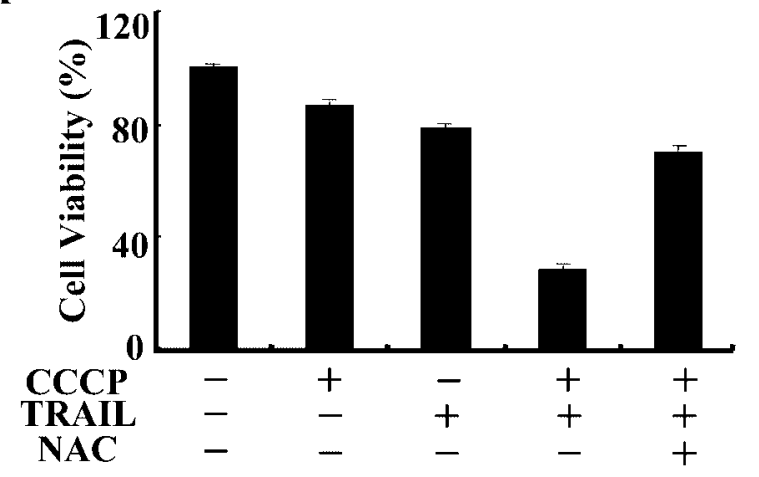

C

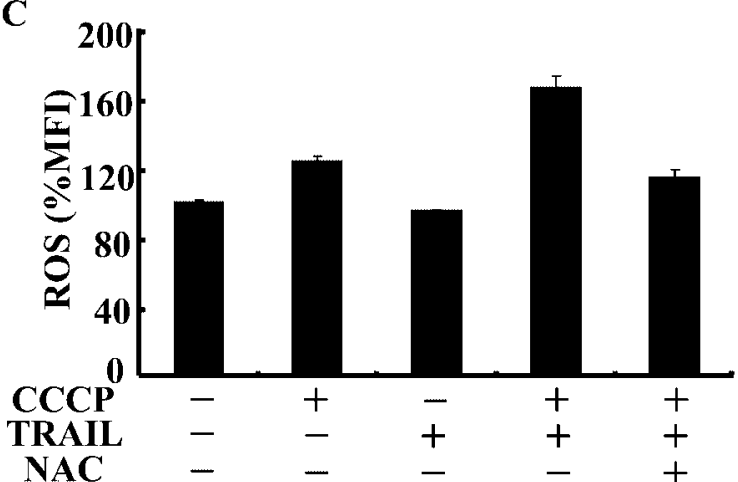

B
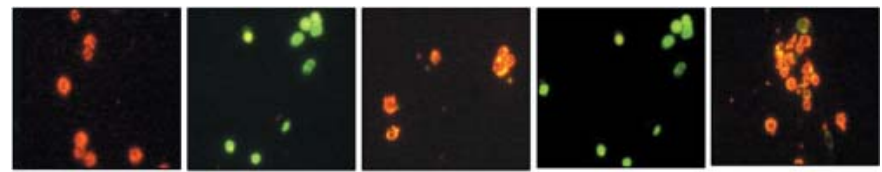

\section{CCCP - \\ TRAIL - \\ NAC -}

+
-

$\overline{+}$

$\begin{array}{ll}+ & + \\ + & + \\ - & +\end{array}$

Fig. 2. Loss of $\triangle \Psi \mathrm{m}$ and ROS generation regulate the enhanced effect of CCCP on TRAIL-induced apoptosis. (A) SNU-638 cells plated on a 12-well plate were treated with CCCP $(20 \mu \mathrm{M})$ for $6 \mathrm{hr}$ and then incubated with or without recombinant TRAIL protein $(100 \mathrm{ng} /$ $\mathrm{m} l$ ) for an additional $3 \mathrm{hr}$. NAC $(1 \mathrm{mM})$ was added $1 \mathrm{hr}$ before CCCP treatment. Cell viability was determined by crystal violet staining method. Viability of control cells was set at $100 \%$, and viability relative to the control was presented. The experiments were performed in triplicate, at least twice. (B) SNU-638 cells plated on a 6-well plate were treated with NAC $(1 \mathrm{mM})$ for $1 \mathrm{hr}$ before CCCP $(20 \mu \mathrm{M})$ treatment for $2 \mathrm{hr}$ and then incubated with or without recombinant TRAIL protein $(100 \mathrm{ng} / \mathrm{m} l)$ for an additional $1 \mathrm{hr}$. The $\Delta \Psi \mathrm{m}$ was determined using a 5,5',6,6'-Tetrachloro-1,1',3,3'-tetraethylbenzimidazol-carbocyanine iodide $(5 \mu \mathrm{M})$ probe. The cells were photographed using a fluoroscope. (C) SNU-638 cells were treated as detailed in A, and ROS generation was measured in media using a fluorescent dye, 2',7'-Dichlorofluorescein diacetate $(10 \mu \mathrm{M})$. The ROS level was measured using spectrofluorometer after incubation for $30 \mathrm{~min}$.

TRAIL treated cells, which corresponds to the cell viability results (Fig. 2A). Thus, CCCP enhanced TRAIL-induced apoptosis by lowering $\triangle \Psi \mathrm{m}$ and ROS generation and thereby activating the Bax and Smac proteins.

\section{DISCUSSION}

The effects of CCCP treatment on TRAIL-induced apoptosis in solid tumors have yet to be clearly defined. It has been reported that CCCP sensitizes tumor cell lines to TRAIL-induced apoptosis by enhancing caspase- 8 and Bax activation and inducing the release of cytochrome-c and Smac into the cytosol $[7,8]$. Therefore, we examined the effect of CCCP on TRAIL-induced cell death in SNU-638 cells, a human gastric cancer cell line. CCCP treatment sensitized the SNU-638 cells to TRAIL-induced apoptosis. Thus, like the leukemia and human colon carcinoma cell lines, CCCP enhances TRAIL-induced apoptosis in SNU638 cells $[7,8]$. There are conflicting reports concerning apoptosis induction by CCCP alone in a variety of cell lines. For example, CCCP alone does not induce apoptosis in human osteosarcoma cell lines [11] and SKW6 cells [12], while it does induce apoptosis in FL5.12 and Jurkat cells [4]. Here, we found that CCCP alone had a low apoptotic effect on the SNU-638 cell line. Moreover, CCCP was found to enhance the apoptosis-inducing ability of Fas/APO-1/CD95 signaling in Jurkat and CEM cells without causing apoptotic changes [12]. In the present study, we also found that CCCP enhanced TRAIL-induced apoptosis in a SNU-638 cell line without inducing apoptosis.

CCCP sensitizes leukemia cell lines to TRAIL-induced apoptosis via the intrinsic apoptotic pathway, i.e, by enhancing the conformational changes in Bax, release of cytochrome-c and activation of caspase-3 [7]. Furthermore, generation of ROS by CCCP has been found to be responsible for regulating TRAIL-induced apoptosis in human colon carcinoma cell lines [8]. This study also examined the role of $\triangle \Psi \mathrm{m}$ and ROS generation on the enhanced effect of CCCP on TRAIL-induced apoptosis. Interestingly, NAC, an antioxidant, restored $\triangle \Psi \mathrm{m}$ after the CCCP and TRAIL treatment, which indicates possible involvement of NAC in inhibiting the dissipation of $\Delta \Psi \mathrm{m}$ induced by treatment with CCCP and TRAIL. NAC also inhibited the dissipation of $\Delta \Psi \mathrm{m}$ induced by CCCP alone. Therefore, this is the first 


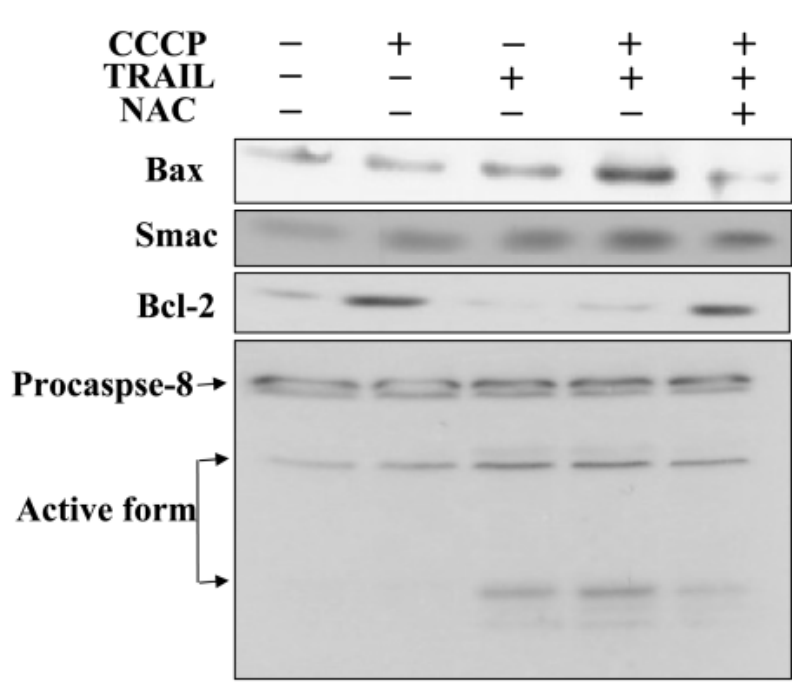

Fig. 3. Effect of CCCP treatment on TRAIL-induced activation of caspases and release of Bax and Smac. SNU-638 cells were treated with CCCP $(20 \mu \mathrm{M})$ for $2 \mathrm{hr}$ and then incubated with or without recombinant TRAIL protein $(100 \mathrm{ng} / \mathrm{m} l)$ for an addi tional $1 \mathrm{hr}$. NAC $(1 \mathrm{mM})$ was added $1 \mathrm{hr}$ before CCCP treatment. Whole cell lysates were prepared and protein sample (40 $\mu \mathrm{g} / \mathrm{m} l)$ were separated on SDS gel and analyzed for apoptotic protein, such as caspase- 8 , Bax, Smac and Bcl-2, by western blotting analysis.

time report showing that NAC, a specific ROS scavenger, might be involved in preventing the CCCP-induced dissipation of the $\Delta \Psi \mathrm{m}$. In the case of human colon carcinoma cells, combined treatment with CCCP and TRAIL abrogated the production of ROS because of complete loss of mitochondrial transmembrane potential. In contrast, in this study, CCCP and TRAIL treatment increased generation of ROS by reducing $\triangle \Psi \mathrm{m}$.

Since CCCP is a mitochondrial poison, it causes reduction $\Delta \Psi \mathrm{m}$ and thereby causes release of apoptotic proteins that lead to apoptosis. Our data suggests that CCCP treatment led to reduction in $\Delta \Psi \mathrm{m}$ and thereby caused release of apoptotic proteins such as Bax, Smac and activated caspase8. Activation of caspase- 8 , Bax and Smac proteins were found to be ROS-dependent. NAC treatment abrogated ROS generation, reduction of $\Delta \Psi \mathrm{m}$ and activation of these proteins. Inhibition of change in $\Delta \Psi \mathrm{m}$ by treatment with NAC suggests that CCCP enhances the effect of TRAIL by first altering $\triangle \Psi \mathrm{m}$, thereby leading to generation of ROS and expression of apoptotic protein. Furthermore, NAC treatment induced expression of Bcl-2 protein, which is an antiapoptotic protein. It was reported that $\mathrm{Bcl}-2$ overexpression protects tumor cells from CCCP induced apoptosis but does not prevent depolarization of mitochondrial transmembrane potential [1]. Similarly, in this study, we found that cells treated with CCCP alone expressed Bcl-2, which indicates that overexpression of $\mathrm{Bcl}-2$ protected the cells from CCCPinduced apoptosis but it did not prevent depolarization of $\triangle \Psi \mathrm{m}$. Moreover, NAC treatment induced expression of the
Bcl-2 protein in CCCP and TRAIL-treated cells. These data suggest that CCCP induced dissipation of $\triangle \Psi \mathrm{m}$ and led to ROS generation and ultimately apoptosis. Herein, we also report that although NAC is an antioxidant, it first inhibits changes in $\triangle \Psi \mathrm{m}$ and thereby inhibits enhacement of the effect CCCP on TRAIL-induced apoptosis.

In conclusion, CCCP enhanced TRAIL-induced apoptosis in a time and dose dependent manner by dissipation of $\triangle \Psi \mathrm{m}$ and ROS generation. Therefore, combined CCCP and TRAIL treatment might be useful in cancer therapy, particularly for TRAIL resistant cells. In addition, uncoupling with CCCP can be used as a successful chemotherapeutic tool for ligand tumor therapy using TRAIL.

ACKNOWLEDGEMENTS. This work was supported by the Regional Research Centers Program of the Korean Ministry of Education \& Human Resources Development through the Center for Healthcare Technology Development and 2nd stage Brain Korea 21 Project.

\section{REFERENCES}

1. Armstrong, J.S., Steinauer, K.K., French, J., Killoran, P.L., Walleczek, J., Kochanski, J. and Knox, S.J. 2001. Bcl-2 inhibits apoptosis induced by mitochondrial uncoupling but does not prevent mitochondrial transmembrane depolarization. Exp. Cell Res. 262: 170-179.

2. Carson, D.A. and Ribeiro, J.M. 1993. Apoptosis and disease. Lancet 341: 1251-1254.

3. Carson, D.A. and Tan, E.M. 1995. Apoptosis in rheumatic disease. Bull. Rheum. Dis. 44: 1-3.

4. De Graaf, A.O., van den Heuvel, L.P., Dijkman, H.B., de Abreu, R.A., Birkenkamp, K.U., de Witte, T., van der Reijden, B.A., Smeitink, J.A. and Jansen, J.H. 2004. Bcl-2 prevents loss of mitochondria in CCCP-induced apoptosis. Exp. Cell Res. 299: 533-540.

5. Ellis, R.E., Yuan, J.Y. and Horvitz, H.R. 1991. Mechanisms and functions of cell death. Annu. Rev. Cell Biol. 7: 663-698.

6. Griffith, T.S., Anderson, R.D., Davidson, B.L., Williams, R.D. and Ratliff, T.L. 2000. Adenoviral-mediated transfer of the TNF-related apoptosis-inducing ligand/Apo-2 ligand gene induces tumor cell apoptosis. J. Immunol. 165: 2886-2894.

7. Hao, J.H., Yu, M., Liu, F.T., Newland, A.C. and Jia, L. 2004. $\mathrm{Bcl}-2$ inhibitors sensitize tumor necrosis factor-related apoptosis-inducing ligand-induced apoptosis by uncoupling of mitochondrial respiration in human leukemic CEM cells. Cancer Res. 64: 3607-3616.

8. Izeradjene, K., Douglas, L., Tillman, D.M., Delaney, A.B. and Houghton, J.A. 2005. Reactive oxygen species regulate caspase activation in tumor necrosis factor-related apoptosisinducing ligand-resistant human colon carcinoma cell lines. Cancer Res. 65: 7436-7445.

9. Jacobson, M.D., Weil, M. and Raff, M.C. 1997. Programmed cell death in animal development. Cell 88: 347-354.

10. Kroemer, G. and Reed, J.C. 2000. Mitochondrial control of cell death. Nat. Med. 6: 513-519.

11. Lim, M.L., Minamikawa, T. and Nagley, P. 2001. The protonophore CCCP induces mitochondrial permeability transition without cytochrome $\mathrm{c}$ release in human osteosarcoma cells. FEBS Lett. 503: 69-74.

12. Linsinger, G., Wilhelm, S., Wagner, H. and Hacker, G. 1999. 
Uncouplers of oxidative phosphorylation can enhance a Fas death signal. Mol. Cell Biol. 19: 3299-3311.

13. Mlejnek, P. 2001. Caspase-3 activity and carbonyl cyanide mchlorophenylhydrazone-induced apoptosis in HL-60. Altern. Lab. Anim. 29: 243-249.

14. Muscarella, D.E., O'Brien, K.A., Lemley, A.T. and Bloom, S.E. 2003. Reversal of Bcl-2-mediated resistance of the EW36 human B-cell lymphoma cell line to arsenite- and pesticideinduced apoptosis by PK11195, a ligand of the mitochondrial benzodiazepine receptor. Toxicol. Sci. 74: 66-73.

15. Nagata, S. 1997. Apoptosis by death factor. Cell 88: 355-365.

16. Newmeyer, D.D. and Ferguson-Miller, S. 2003. Mitochondria: releasing power for life and unleashing the machineries of death. Cell 112: 481-490.

17. O'Brien, K.A., Muscarella, D.E. and Bloom, S.E. 2001. Differential induction of apoptosis and MAP kinase signaling by mitochondrial toxicants in drug-sensitive compared to drugresistant B-lineage lymphoid cell lines. Toxicol. Appl. Pharmacol. 174: 245-256.

18. Park, S.Y., Billiar, T.R. and Seol, D.W. 2002. Hypoxia inhibition of apoptosis induced by tumor necrosis factor-related apoptosis-inducing ligand (TRAIL). Biochem. Biophys. Res. Commun. 291: 150-153.
19. Pitti, R.M., Marsters, S.A., Ruppert, S., Donahue, C.J., Moore, A. and Ashkenazi, A. 1996. Induction of apoptosis by Apo-2 ligand, a new member of the tumor necrosis factor cytokine family. J. Biol. Chem. 271: 12687-12690.

20. Raff, M.C. 1996. Size control: the regulation of cell numbers in animal development. Cell 86: 173-175.

21. Seol, D.W. and Billiar, T.R. 1999. A caspase-9 variant missing the catalytic site is an endogenous inhibitor of apoptosis. $J$. Biol. Chem. 274: 2072-2076.

22. Walczak, H., Miller, R.E., Ariail, K., Gliniak, B., Griffith, T.S., Kubin, M., Chin, W., Jones, J., Woodward, A., Le, T., Smith, C., Smolak, P., Goodwin, R.G., Rauch, C.T., Schuh, J.C. and Lynch, D.H. 1999. Tumoricidal activity of tumor necrosis factor-related apoptosis-inducing ligand in vivo. Nat. Med. 5: 157 163.

23. Wiley, S.R., Schooley, K., Smolak, P.J., Din, W.S., Huang, C.P., Nicholl, J.K., Sutherland, G.R., Smith, T.D., Rauch, C., Smith, C.A. and et al. 1995. Identification and characterization of a new member of the TNF family that induces apoptosis. Immunity 3: 673-682.

24. Wyllie, A.H., Kerr, J.F. and Currie, A.R. 1980. Cell death: the significance of apoptosis. Int. Rev. Cytol. 68: 251-306. 